\title{
Efficient gene targeting in mouse zygotes mediated by CRISPR/Cas9-protein
}

\author{
Chris J. Jung • Junli Zhang • Elizabeth Trenchard • Kent C. Lloyd • David B. West • \\ Barry Rosen • Pieter J. de Jong (i)
}

Received: 17 June 2016/Accepted: 10 November 2016/Published online: 30 November 2016

(C) The Author(s) 2016. This article is published with open access at Springerlink.com

\begin{abstract}
The CRISPR/Cas9 system has rapidly advanced targeted genome editing technologies. However, its efficiency in targeting with constructs in mouse zygotes via homology directed repair (HDR) remains low. Here, we systematically explored optimal parameters for targeting constructs in mouse zygotes via HDR using mouse embryonic stem cells as a model system. We characterized several parameters, including single guide RNA cleavage activity and the length and symmetry of homology arms in the construct, and we compared the targeting efficiency between Cas9, Cas9nickase, and dCas9-FokI. We then applied the optimized conditions to zygotes,
\end{abstract}

Electronic supplementary material The online version of this article (doi:10.1007/s11248-016-9998-5) contains supplementary material, which is available to authorized users.

C. J. Jung · D. B. West · P. J. de Jong $(\square)$

University of California, San Francisco Benioff

Children's Hospital Oakland Research Institute, Oakland,

CA 94609, USA

e-mail: pdejong@chori.org

J. Zhang

Gladstone Institutes, San Francisco, CA 94158, USA

E. Trenchard $\cdot$ B. Rosen

Wellcome Trust Sanger Institute, Cambridge CB10 1SA, UK

K. C. Lloyd

Mouse Biology Program, University of California, Davis, CA 95618, USA delivering Cas9 as either mRNA or protein. We found that Cas9 nucleo-protein complex promotes highly efficient, multiplexed targeting of circular constructs containing reporter genes and floxed exons. This approach allows for a one-step zygote injection procedure targeting multiple genes to generate conditional alleles via homologous recombination, and simultaneous knockout of corresponding genes in nontargeted alleles via non-homologous end joining.

Keywords CRISPR - Transgenic mouse model · Gene targeting

\section{Introduction}

Technologies enabling efficient and precise genome editing render powerful tools for studying biology, and open new avenues for explorative endeavors in biomedicine and translational research. Until recently, genome engineering in cell and animal models relied on random mutagenesis, random insertion of transgenes, or inefficient targeting, which greatly limited scientific progress (Stanford et al. 2001; Yu and Bradley 2001; Austin et al. 2004; Gondo 2008). Over the past decade, genome editing technologies have undergone a rapid procession of improvements in efficiency and precision with the development of zinc finger nucleases (ZFNs) (Kim et al. 1996; Bibikova et al. 2003; Maeder et al. 2008), and transcription 
activator-like effector nucleases (TALENs) (Christian et al. 2010; Boch 2011; Cermak et al. 2011). These tools are based on customizable DNA binding modules attached to nucleases for targeted chromosome breaks. More recently, the clustered regularly interspaced short palindromic repeats (CRISPR) associated protein 9 (Cas9) has emerged with great potential. In contrast to ZFNs and TALENs, which depend on protein-DNA interactions, the CRISPR/Cas9 system is based on the principle of engineering a single guide RNA (sgRNA) for base pairing with complementary DNA sequences for site-specific cleavage by the associated Cas9 protein complex (Gaj et al. 2013; Mali et al. 2013a, b; Sander and Joung 2014; Jiang and Marraffini 2015).

The inherent simplicity and flexibility imbued in the CRISPR/Cas9 architecture has propelled the system as the ideal genome engineering tool (Horvath and Barrangou 2010; Marraffini and Sontheimer 2010; Jinek et al. 2012; Wiedenheft et al. 2012; Cong et al. 2013; Mali et al. 2013a, b). As such, the system has been particularly useful for applications aimed at direct or conditional knockout of gene functions. For example, reports have shown that stimulating the error-prone mechanism of nonhomologous end joining (NHEJ) repair (Rouet et al. 1994) by the sgRNA:Cas9 complex induced DNA breaks can knockout gene function by creating indel mutations (Cho et al. 2013; Shen et al. 2013; Wang et al. 2013; Sung et al. 2014) and that injecting single-strand oligonucleotides (ssODNs) carrying loxP sequences or short tags into zygotes can generate conditional alleles (Yang et al. 2014; Yoshimi et al. 2014; Renaud et al. 2016). However, despite the growing body of literature supporting the ease with which transgenic animals can be generated with the CRISPR/Cas9 system, approaches based on NHEJ or genome modification using ssODNs, suffer from imprecise NHEJ dependent genome modification, or short cargo carrying capacity and trans allele effect.

While using constructs may overcome these limitations, their low targeting efficiency with the CRISPR/Cas9 system hinders robust high-throughput applications. To date, only a few reports have described methods to knock small constructs into mouse zygotes with the CRISPR/Cas9 system. For example, Yang et al. (2013) injected circular reporter plasmids (Nanog-mCherry or Oct4-GFP) carrying homology arm lengths between 2 and $4.5 \mathrm{kbp}$ with a targeting efficiency of approximately $10 \%$, while Chu et al. (2016) targeted the Rosa26 locus using vectors carrying asymmetric homology arm lengths between 1 and $4 \mathrm{kbp}$ with a targeting efficiency of 0-20\%. Moreover, Aida et al. (2015) described increased targeting efficiency of a circular EGFP-reporter vector with 2 kbp homology arms using Cas9 protein combined with chemically synthesized dual-crRNA:tracrRNA; however, their experiments were unsuccessful when using only the Cas9 protein. In contrast, Menoret et al. (2015) reported successful targeting using Cas 9 protein and a linearized podocanneoR cassette with 1 and $4.2 \mathrm{kbp}$ asymmetric homology arms. Others reported success based on a singletargeted founder (F0) pup. Indeed, Wang et al. (2015) used a single-injection experiment to target 1 of 16 founder pups with a Cre cassette containing approximately 600 bp homology arms, and Lee and Lloyd (2014) used a single-injection in zygotes to successfully target 1 of 13 founder pups with a cassette containing a floxed critical exon with $1.9 \mathrm{kbp}$ homology arms digested out of a circular vector. While these reports provide some insight, the scarcity of literature and the lack of protocol standards highlight a need to further optimize these methods and test their reliability.

Of particular relevance is the existence of more than 15,000 custom reporter vectors for conditional knockout are available to the public through repositories created by the Knockout Mouse Project (KOMP) Resource Center and the European Conditional Mouse Mutagenesis (EUCOMM) Center. This multi-center collaborative effort aims to ascribe the function of the entire mouse genome (Skarnes et al. 2011; Bradley et al. 2012). Despite these resources, the process of generating transgenic mouse models remains slow, because we lack an efficient and reliable method to target these constructs in mouse zygotes. As a result, many research facilities continue to rely on the traditional method of using ES cells to generate transgenic mouse models, which is cumbersome and inefficient (Capecchi 2005).

In this study, we aimed to develop an optimized condition for HDR mediated construct targeting in mouse zygotes using CRISPR/Cas9. First, we randomly selected constructs from the KOMP/ EUCOMM repository that included small deletions of non-essential intronic sequences separating 
"critical exons" from upstream and downstream homology arms, which made them particularly useful for targeting studies. We then designed sgRNAs for these targets, which allowed correctly targeted genomic sites to resist further cutting by the Cas9:sgRNA complex. A representative sample of vectors was selected for in-depth analysis using embryonic stem (ES) cells as a model system for determining the optimal parameters for HDR-mediated targeting, which included comparing Cas9, Cas9nickase (Cas9n), and catalytically inactive Cas9 fused to FokI endonuclease (dCas9-FokI), and varying the length and symmetry of the homology arms. We applied the optimized conditions to zygotes and delivered Cas9 as either mRNA or protein to further hone and enhance the parameters. With our systematic approach for defining the optimal targeting conditions, we showed that Cas 9 protein promotes an efficient multiplexed targeting of circular constructs containing reporter genes and floxed exons, and that this approach supports a one-step procedure to inject zygote to achieve both HDR mediated targeting of multiples genes and NHEJ induced deletion of gene function. Hence, we provide a blueprint describing an efficient and reliable method for CRISPR/Cas9 mediated construct targeting in mouse zygotes.

\section{Results}

Strategy for designing sgRNAs to mediate KOMP construct targeting in mouse ES cells

To investigate whether CRISPR/Cas9(n) mediation enhances targeting of KOMP constructs in ES cells, we randomly selected vector constructs for nine genes from the KOMP repository (Fig. S1a-i). We designed two sgRNAs (A-sgRNA and B-sgRNA) as matched pairs for critical exon sequences in each of the nine genes, which would allow double-nicking with the Streptococcal pyogenes (Sp) Cas9 nickase mutant D10A (Ran et al. 2013), hereafter referred to as "Cas9n" (Fig. 1a, b). One of the sgRNAs (A-sgRNA) of each pair was used with the wild-type SpCas9 to generate blunt-ended DNA cuts. All sgRNAs were designed using the CRISPR guide-design tool developed by the Zhang lab at MIT (crispr.mit.edu) (Hsu et al. 2013).
In vitro and ex vivo evaluation of sgRNA activity

To verify the efficiency of individual sgRNAs, we designed two assays to evaluate the capacity of selected sgRNAs to cleave dsDNA by wild-type Cas9 or double-nick opposite strands by Cas9n. First, we designed an in vitro approach in which the sgRNA target sequences were cloned into a plasmid that would be linearized at a unique restriction site in the vector backbone (Fig. S2a). Each linearized vector was incubated with the corresponding A-sgRNA and Cas9 protein, which produced two fragments when cleaved. Using this approach, we observed highly efficient dsDNA-cutting activity for all but two sgRNAs (i.e., Ano3 and Cesld), which showed relatively low efficiency (Fig. 1c). In the second assay, we designed an ex vivo approach to evaluate sgRNA activity in a mammalian cell culture system. Specifically, with a single-strand annealing (SSA) approach, we measured the capacity of single sgRNAs or matched sgRNA pairs to catalyze homologous recombination within a transfected plasmid, repairing a non-functional luciferase gene. The sgRNA target sequences were cloned in between direct repeats of the first $300 \mathrm{bp}$ of the firefly luciferase open reading frame (ORF), with the upstream repeat having a Ubc promoter and the downstream $300 \mathrm{bp}$ connected to the remaining ORF sequence. Cuts induced by Cas 9 or matched nicks induced by Cas9n can stimulate homologous recombination between the repeats to generate a functional luciferase, measured by fluorescence (Fig. 1e). In this assay, the sgRNA target plasmid, expressing the sgRNA under the mammalian PGK promoter, and a Cas9- or Cas9n-expressing plasmid were co-transfected into T293 cells. Human adeno-associated virus integration site 1 (AAVS1) sgRNA with a robust genome-editing capability was used as a positive control (Mali et al. 2013). The SSA assay revealed that all sgRNAs that were highly active in the in vitro assay [Rnf10, Nalcn, Dnase1/2(E2), Dnase 1/2(E7), Ap4e1, Nxn, Dbn1, and Asic4] induced the expression of firefly luciferase to levels comparable to the AAVSI control; however, the two sgRNAs with less in vitro activity also showed somewhat lower activity in the transfection assay (Fig. 1f). Therefore, these methods provide a simple approach for evaluating the activity of sgRNAs and show that most of the sgRNAs designed from a randomly selected list of 
A
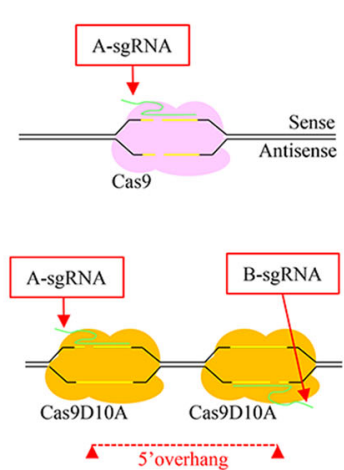

C

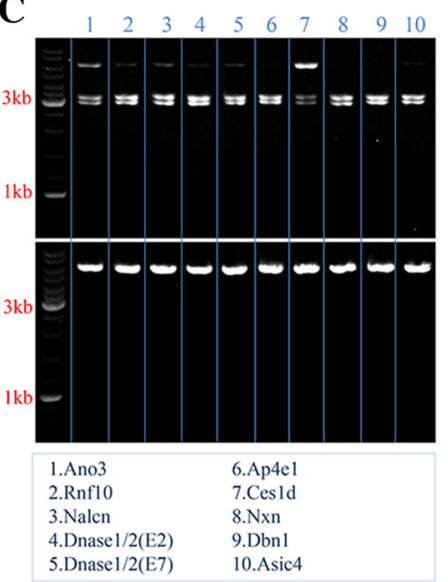

D

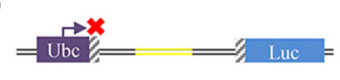

Cas $9+$ gRNA bind to target DNA sequence

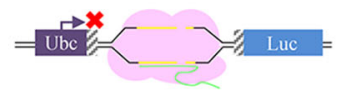

dsDNA break induces SSA repair

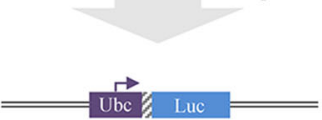

B

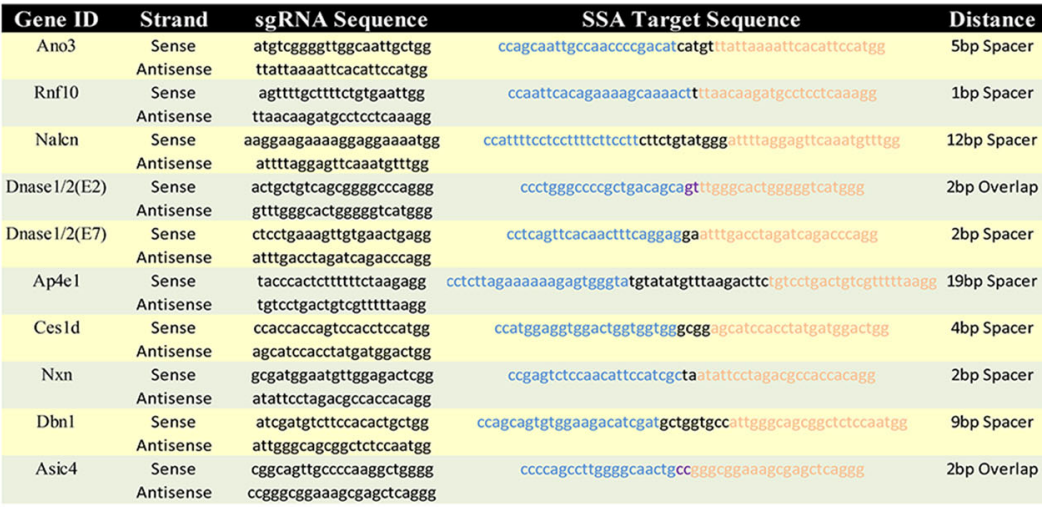

E

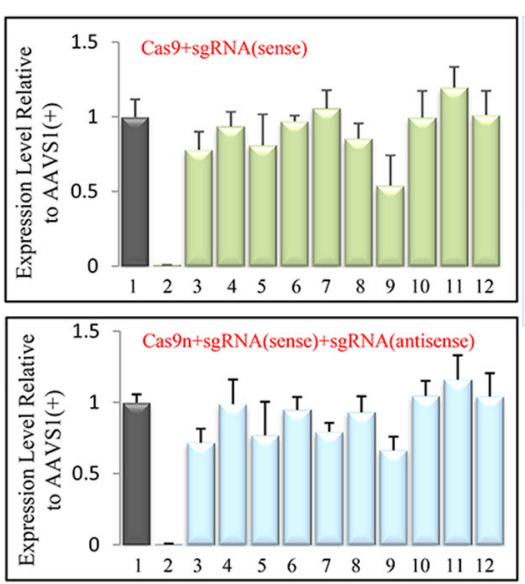

Fig. 1 Strategy for CRISPR/Cas9(n) mediated KOMP construct targeting. a Diagram illustrating sgRNA-design strategy for use with Cas9 and/or Cas9n. The top illustration describes a single sgRNA:Cas9-complex strategy for inducing dsDNA cleavage. The bottom illustration describes a scenario in which a pair of sgRNA:Cas9n complexes are combined to induce nicks on opposite DNA strands, creating $5^{\prime}$ overhangs if the paired sgRNA:Cas9n complexes are not overlapping (the overlapping complexes form $3^{\prime}$ overhangs). Green, sgRNAs. b Table listing the sgRNAs selected for each gene in (a) (first column), the strand to which each sgRNA was designed to bind (second

genes are active at levels comparable to the highly active AAVSI sgRNA.

CRISPR/Cas9(n) mediated targeting of KOMP constructs in mouse ES cells

Next, we chose one of the genes with sgRNA activity most similar to that of sgRNA-AAVS1, Nxn, for an indepth analysis to optimize the parameters for construct targeting stimulated by Cas9(n) in mouse ES cells. The Nxn KOMP vector has a multifunctional lacZ reporter and a preconditional "knockout-first" design (Testa et al. 2004; Skarnes et al. 2011) with 3.6 kbp 5' column), sgRNA sequences (third column), sgRNA-target sequences (blue, sgRNA target on the sense strand; red, sgRNA target on the antisense strand; black, spacer sequence between the two targets) (forth column), and spacer distance (last column). c Agarose analysis showing result of in vitro assay with Cas9 protein. d Schematic diagram illustrating the mechanism of the SSA assay. e Bar graph showing the SSA assay result relative to the AAVS1 sgRNA. Top graph, result using Cas9; bottom graph, result using Cas9n. AAVS1 (-) indicates the negative control, which is identical to AAVS1 (+) without sgRNA. (Color figure online)

and $3.8 \mathrm{kbp} 3^{\prime}$ homology arms. This design was created for a linearized format with a diphtheria toxin A (DTA) cassette for negative selection to favor homologous targeting over random integration (Fig. 2a). For Cas9-stimulated targeting, the original vector (pKOMP-Nxn, $17.9 \mathrm{kbp}$ ) was converted into a derivative that lacks DTA, to use as a circular construct for targeting. The homology arms flank an FRT-encased promoterless lacZ reporter followed by a neomycin/G418-resistant gene that relies on a floxed critical exon and is controlled by an endogenous promoter. To determine whether Cas9(n) enhances targeting efficiency, plasmids expressing A-sgRNA- 
A

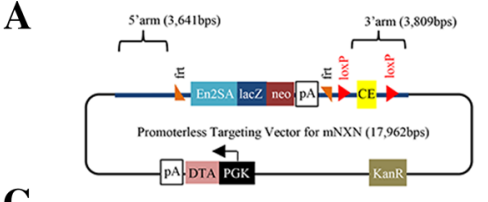

C

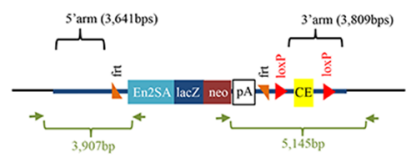

D

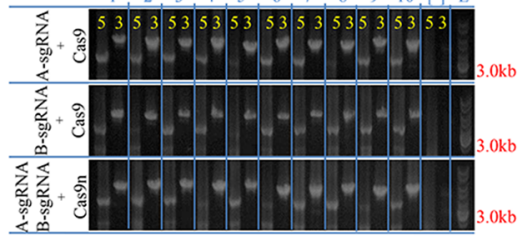

F

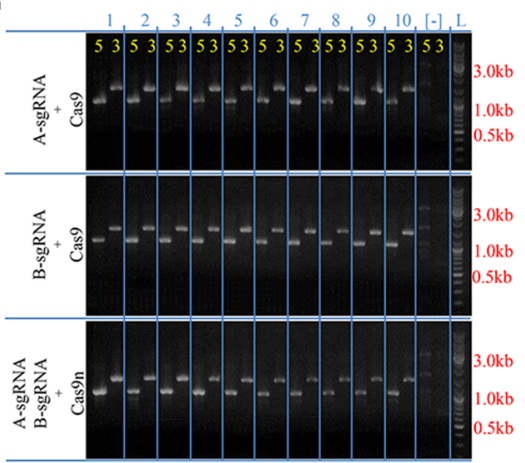

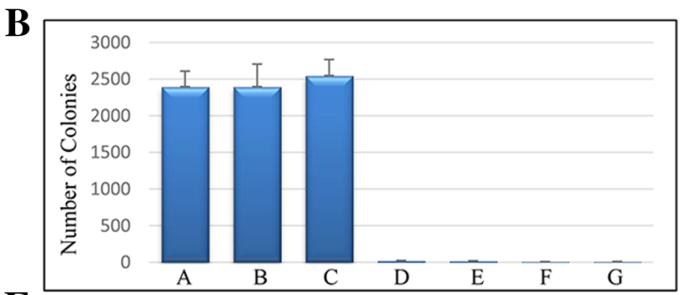
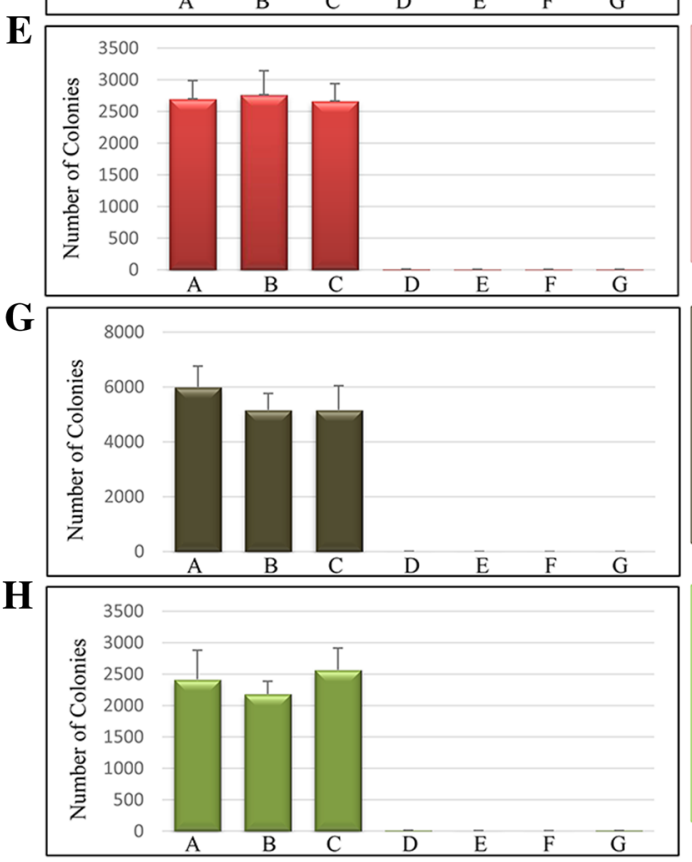

pKOMP- $N x n$

A. A-sgRNA-N $x n+$ Cas 9 B. B-sgRNA-N $x n+$ Cas 9

C. A-sgRNA-Nxn+

B-sgRNA-N $x n+\operatorname{Cas} 9 n$

D. Cas9

Cas9n

F. A-sgRNA- $N x n+$ Cas9n

G. B-sgRNA- $N x n+\operatorname{Cas} 9 n$

\section{pKOMP- $N x n-900$}

A. A-sgRNA-N $x n+$ Cas 9 B. B-sgRNA-Nxn+ Cas

C. A-sgRNA-Nxn+

B-sgRNA-N $N n+$ Cas9n

D. Cas 9

E. Cas 9 n

. A-sgRNA- $N x n+$ Cas $9 n$

G. B-sgRNA-Nxn+Cas9n

\section{DKOMP-DbnI-900}

A. A-sgRNA- $\mathrm{N} x n+\mathrm{Cas} 9$ B. B-sgRNA-N $x n+$ Cas

C. A-sgRNA- $N x n+$
B-sgRNA- $N x n+$ Cas9n

B-sg

D. Cas9
E. Cas9n

F. A-sgRNA-Nxn+Cas9n G. B-sgRNA- $N x n+\operatorname{Cas} 9 n$

\section{pKOMP-Asic4-900}

A. Sense-sgRNA- $\mathrm{N} x n+\mathrm{Cas} 9$ B. Antisense-sgRNA- $N x n+$ Cas9 C. Sense-sgRNA-Nxn+

Antisense-sgRNA- $\mathrm{N} x n+\mathrm{Cas} 9 \mathrm{n}$

D. Cas9

D. Cas9
E. Cas9n

F. Sense-sgRNA- $N x n+$ Cas9n

G. Antisense-sgRNA- $N x n+$ Cas9n
Fig. 2 Cas9(n)-mediated targeting efficiency of constructs with long and short homology arms. a pKOMP-Nxn construct diagram. The promoterless construct was designed such that the lacZ reporter and neomycine/G418-resistance gene were controlled by the endogenous $N x n$ promoter upon proper targeting. The critical exon (CE) was flanked by loxP sequences, therefore, the transgenic mouse derived from this construct can be converted into either a conditional or reporter-marked knockout. b Bar graph showing the number of G418-resistant ES cell colonies post-electroporation with plasmids expressing sgRNA and Cas9(n), along with the unmodified pKOMP-Nxn construct. The results show an increase in the number of colonies in the Cas9(n) mediated conditions (" $A$ ", " $B$ ", and " $C$ "). Conditions with Cas9(n) and pKOMP-Nxn without sgRNA(s), and sense- or antisense-sgRNA-Nxn with Cas9n and $\mathrm{pKOMP}-N x n$, were used as negative controls (" $D$ ", " $E$ ", " $F$ ", and " $G$ "). c Diagram illustrating the junction PCR method

$N x n$ and Cas9 were co-transfected into mouse ES cells with either pKOMP-Nxn or pKOMP-Nxn-del that lacked the floxed exon via Cre recombinase (Fig. S3a). Our post-transfection results revealed more than a 2000-fold increase in G418-resistant colonies relative to negative controls, and the PCR analysis of randomly selected colonies showed more than $90 \%$ to have for verifying correct targeting with products of $3907 \mathrm{bp}\left(5^{\prime} \mathrm{arm}\right)$ and $5145 \mathrm{bp}\left(3^{\prime} \mathrm{arm}\right)$. d Junction PCR results for 10 random colonies selected from the G418-resistant colonies. The top row shows PCR products from 10 random colonies selected from electroporation using plasmids expressing A-sgRNA-Nxn and Cas9, along with pKOMP-Nxn. The middle row shows B-sgRNA-Nxn and Cas9, along with pKOMP-Nxn. The bottom row shows A-sgRNA-Nxn, B-sgRNA-Nxn and Cas9n, along with pKOMP-Nxn. (-), negative control. e Same bar graph as (b) except using the pKOMP-Nxn-900 targeting construct. f Same PCR as (d) except based for clones generated by the pKOMP-Nxn-900 targeting construct. The expected PCR products are 1105 bp ( $5^{\prime}$ junction) and 1463 ( $3^{\prime}$ junction). (-), negative control. g Same bar graph as (b) except using the pKOMP-Dbn1-900 targeting construct (Fig. 1a). h Same bar graph as (b) except using the pKOMP-Asic4-900 targeting construct (Fig. 1a)

constructs correctly targeted in the genome (Fig. 2bd). The pKOMP-Nxn-del vector showed slightly higher targeting success, whereas the pKOMP-Nxn vector showed infrequent clones negative for the $3^{\prime}$ loxP PCR, likely resulting from HDR between the $5^{\prime}$ arm and the critical exon. Sequence analysis of the PCR products and copy number analysis using RT- 
qPCR confirmed the integrity of the targeting (data not shown). We repeated the experiment by co-transfecting plasmids expressing B-sgRNA- $N x n$ with Cas9 or the matched sgRNA-Nxn pair with Cas9n, which produced similarly high targeting efficiency (Fig. 2bd). These observations indicate that sgRNA mediated stimulation of homologous recombination by either a dsDNA break with Cas 9 or paired double-nicking with Cas9n enhances pKOMP-Nxn targeting efficiency in mouse ES cells. We also tested for the presence Cas9 plasmid integration in 10 targeted clones and found them to be absent (Fig. S3b).

Reduction of homology arm length to $900 \mathrm{bp}$ does not effect CRISPR/Cas9(n) mediated targeting efficiency

In designing the KOMP vector (Skarnes et al. 2011), we kept the homology arms larger than $3 \mathrm{kbp}$ to maximize targeting efficiency in mouse ES cells. In light of Cas9(n) increasing the efficiency, we explored how decreasing the length of the homology arms affected targeting. With a single-step gap-repair approach, we shortened the pKOMP-Nxn homology arms to 900 bp (pKOMP-Nxn-900). Then we transfected this modified vector into mouse ES cells with plasmids expressing Cas9 and A-sgRNA-Nxn; Cas9 and B-sgRNA-Nxn; or Cas9n, A-sgRNA-Nxn, and B-sgRNA-Nxn. Post-transfection, we observed a high number of G418-resistant colonies in all three conditions, similar to transfection with the non-deleted pKOMP-Nxn vector (Fig. 2e). Junction PCR analysis of 10 randomly selected colonies from each of the Cas9(n)-mediated conditions confirmed correct targeting in all of the analyzed samples (Fig. 2f). Analysis of different KOMP vectors modified with 900 bp homology arms, pKOMP-Dbn1-900 and pKOMP-Asic4-900, produced similarly high targeting efficiency (Fig. 2g, h). These observations indicated that Cas9(n) greatly enhances targeting of KOMP vectors in mouse ES cells, and that reducing the homology arm lengths to $900 \mathrm{bp}$ did not compromise the efficiency.

FokI nuclease fused to catalytically inactive Cas9 decreases targeting efficiency

Recently, Guilinger et al. (2014) and Tsai et al. (2014) used dCas9-FokI in a dimeric form to reduce non- specific genome editing. They reasoned that the obligate dimeric form of dCas9-FokI would cleave the DNA only when two distinct dCas9-Fok1:sgRNA complexes simultaneously bound to adjacent sites with particular spacing constraints (Fig. S4a). While interesting, whether dCas9-FokI can induce HDR for efficient construct targeting remains unknown. Specifically, the stringent spatial requirements for assembling dCas9-FokI dimers and the bulky hybrid protein may affect the efficiency of construct targeting.

We sought to compare the targeting efficiencies of pKOMP-Nxn-900 mediated by Cas9n (Fig. S4b) and dCas9-FokI (Fig. S4a). We designed a new FokIsgRNA-Nxn as an obligate dimer with the A-sgRNA$N x n$, such that a 24 bp spacer region separated the two sgRNA-binding sequences (Fig. S4a); the $24 \mathrm{bp}$ distance was determined based on a report by Guilinger et al. (2014) in which a $\sim 15$ or $\sim 25 \mathrm{bp}$ spacer distance between the dimeric sgRNA:dCas9FokI was optimal for gene modification. Our findings show that co-transfecting four plasmids expressing A-sgRNA-Nxn, FokI-sgRNA-Nxn, and Cas9n, along with pKOMP-Nxn-900, reduced the number of G418resistant colonies to approximately half of those observed in conditions where B-sgRNA-Nxn was used instead of FokI-sgRNA-Nxn (Fig. S4c). When the same transfection conditions were repeated to replace Cas9n with dCas9-FokI, the number of G418-resistant colonies reduced to a similar level as the negative controls (Fig. S4c). These observations suggested that while obligate dimeric dCas9-FokI may reduce offtarget activities of sgRNAs, the low efficiency of construct targeting must be improved.

Homology arm length and symmetry are critical for Cas9(n) mediated construct targeting

Next, we wondered whether we could further decrease the homology arm length without ramifications. We reduced the homology arm lengths of pKOMP-Nxn to 500, 250, 120, and 0 bp (Fig. 3a) and transfected the modified targeting constructs with plasmids expressing Cas9 and A-sgRNA-Nxn; Cas9 and B-sgRNA$N x n$; and Cas9n, A-sgRNA-Nxn, and B-sgRNA-Nxn. We observed about a four-fold drop in the number of colonies upon shortening from $900 \mathrm{bp}$ to $500 \mathrm{bp}$ and further proportional decreases with shorter arm lengths of 259 and $125 \mathrm{bp}$ (Fig. 3b). Because these findings indicated that the homology arm lengths must 

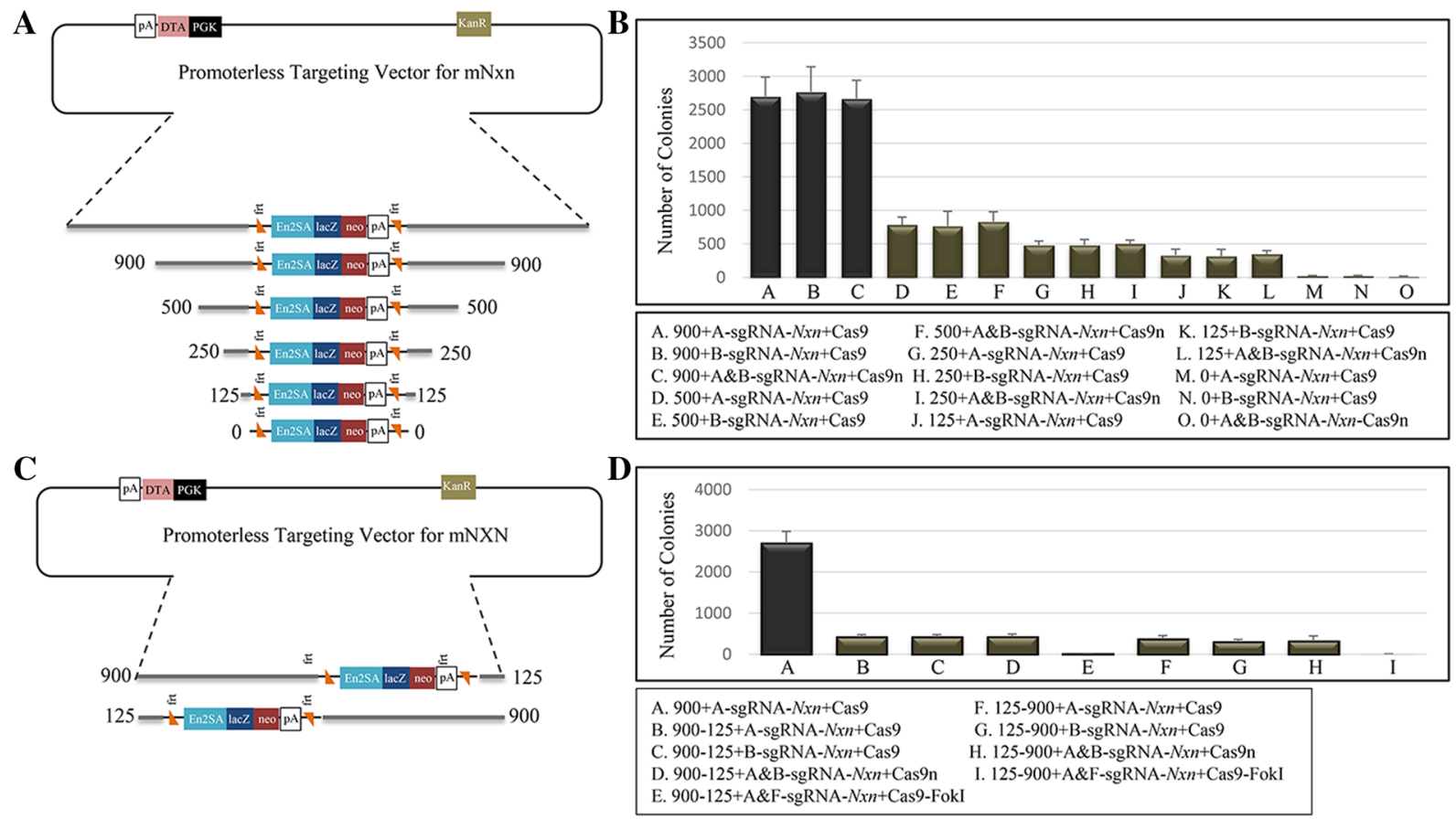

Fig. 3 Homology arm length and symmetry influence targeting efficiency. a Diagram illustrating pKOMP-Nxn constructs with various symmetrical homology arm lengths. b Bar graph showing the number of G418-resistant colonies post-electroporation with pKOMP-Nxn with five different homology arm lengths $(900,500,250,125$, and 0 bps). c Diagram illustrating

be close to $900 \mathrm{bp}$ to maintain high targeting efficiency, we examined whether a single $900 \mathrm{bp}$ arm would suffice. We thus modified the pKOMP-Nxn vector to have a $900 \mathrm{bp}$ homology arm at one end and a $125 \mathrm{bp}$ arm at the other end (Fig. 3c). We transfected these constructs with plasmids expressing Cas9, Cas9n, or dCas9-FokI, along with the corresponding sgRNAs, which revealed that modifying the targeting constructs with asymmetric homology arms drastically decreased the number of G418-resistant colonies to a level comparable to constructs having both arm lengths at $125 \mathrm{bp}$. Thus, constructs with dual homology arms of at least $900 \mathrm{bp}$ must be used to obtain high targeting efficiency.

Cas9 protein yields higher targeting efficiency in mouse zygotes than Cas9 mRNA

Next, we focused on construct targeting in mouse zygotes with the same approach used in ES cell transfection. We
pKOMP-Nxn constructs with asymmetrical homology arm lengths. d Bar graph showing the number of G418-resistant colonies post-electroporation with pKOMP-Nxn carrying asymmetrical homology arm lengths. F-sgRNA-Nxn= FokIsgRNA- Nxn

selected one of the KOMP vectors used in previous ES cell transfection experiments (pKOMP-Asic4-900) for targeting in zygotes. In an initial experiment, we coinjected the pKOMP-Asic4-900 vector with either Cas9 protein or mRNA and A-sgRNA-Asic4. Injections with Cas9 protein (Table 1) produced one out of two founder pups with targeted Asic4 integration (50\%), while injections with Cas9 mRNA produced zero out of four founder pups $(0 \%)$ targeted. This experiment was repeated using a construct with longer ( $2 \mathrm{kbp}$ ) homology arms, which produced 6 out of 17 pups with targeted integration for the Cas9 protein (35\%), as compared to 1 out of 28 for Cas 9 mRNA (4\%). Random selection of one of the founder pups for mating showed that the transgenes were germline transmissible, segregating according to Mendelian genetics ( 8 of $12 \mathrm{~F} 1$ pups were heterozygotes) (Fig. S5). These findings indicate that Cas9 protein is more efficient than Cas9 mRNA at stimulating HDR, thereby increasing the rate of construct targeting via homologous recombination. 
Table 1 CRISPR Cas9 mRNA versus Cas9 protein mediated targeting of KOMP construct in mouse zygotes

\begin{tabular}{|c|c|c|c|c|c|c|c|c|c|}
\hline $\begin{array}{l}\text { Targeting } \\
\text { vector ID }\end{array}$ & $\begin{array}{l}\text { Cas9 } \\
\text { mRNA } \\
\text { versus } \\
\text { protein }\end{array}$ & $\begin{array}{l}\text { Cas9 } \\
(\mathrm{ng} / \\
\mu \mathrm{l})\end{array}$ & $\begin{array}{l}\text { sgRNA } \\
(\mathrm{ng} / \mu \mathrm{l})\end{array}$ & $\begin{array}{l}\text { Targeting } \\
\text { vector }(\mathrm{ng} / \\
\mu \mathrm{l})\end{array}$ & $\begin{array}{l}\# \\
\text { Embryos } \\
\text { transferred }\end{array}$ & $\begin{array}{l}\text { \# Of } \\
\text { pups }\end{array}$ & $\begin{array}{l}\text { Genotype } \\
\text { positive }\end{array}$ & $\begin{array}{l}\% \text { Positive relative to } \\
\text { \# of embryos } \\
\text { transferred }\end{array}$ & $\begin{array}{l}\% \text { Positive } \\
\text { relative to \# of } \\
\text { pups }\end{array}$ \\
\hline $\begin{array}{l}\text { pKOMP- } \\
\text { Asic4 }\end{array}$ & mRNA & 20 & 10 & 20 & 81 & 4 & 0 & 0 & 0 \\
\hline $\begin{array}{c}\text { (900 bp } \\
\text { HAs) }\end{array}$ & Protein & 50 & 25 & 20 & 52 & 2 & 1 & 2 & 50 \\
\hline $\begin{array}{l}\text { pKOMP- } \\
\text { Asic4 }\end{array}$ & mRNA & 20 & 10 & 10 & 78 & 28 & 1 & 1 & 4 \\
\hline $\begin{array}{c}\text { (2000 bp } \\
\text { HAs) }\end{array}$ & Protein & 50 & 25 & 10 & 51 & 17 & 6 & 12 & 35 \\
\hline
\end{tabular}

Strategy for CRISPR/Cas9 mediated deletion of critical exons and multi-vector targeting through a single zygote injection experiment

Thus far, we have shown that sgRNA:Cas9(n) complexes designed to induce DNA cleavage in the critical exon sequence stimulate highly efficient targeting of KOMP vectors with symmetrical homology arms as short as $900 \mathrm{bp}$. In this strategy, additional mutant alleles with disrupted gene function may result from insertions/deletions (indels) in the coding sequence generated by imprecise break repair by the NHEJ pathway (Ran et al. 2013). However, NHEJ events are unpredictable and may produce unexpected splicing and/or frame shifts. Thus, we shifted our strategy toward creating dual breaks that flank coding exons, such that alleles lacking construct integration may, instead, be subject to critical exon deletion via NHEJ, resulting in two types of easily characterized mutations.

Recently, Zhou et al. (2014) showed that chromosomal DNA larger than $100 \mathrm{kbp}$ can be deleted using multiple sgRNA:Cas9 complexes flanking the targeting DNA segment. Thus, we aimed to evaluate whether a pair of sgRNAs targeting regions flanking the critical exon could knock-in the conditional construct through homologous recombination, or alternatively, to delete the entire critical exon. To test the viability of this strategy, we designed two sgRNAs that target regions flanking the critical exon of the Nxn gene. We planned to use these constructs with pKOMP-Nxn-900, such that the guide RNA target sequences were eliminated by either targeted construct integration or a deletion between the up- and downstream cleavage sites (Fig. S6a) (hereafter referred to as Upstream-sgRNA-Nxn-CE and Downstream-sgRNA- $N x n$-CE). The dsDNA cleavage efficiencies of the sgRNAs were validated using the SSA firefly luciferase assay (Fig. 1e) to ensure that their activities were comparable to that of sgRNA-AAVS1, A-sgRNA-Nxn, and B-sgRNA-Nxn (Fig. S6b). We then co-transfected plasmids expressing UpstreamsgRNA-Nxn-CE, Downstream-sgRNA-Nxn-CE, and Cas9, along with pKOMP-Nxn-900, which revealed that the number of G418-resistant colonies was similar to that of A-sgRNA-Nxn and Cas9 and A-sgRNA-Nxn, B-sgRNA-Nxn, and Cas9n (Fig. S6c). Junction PCR results of 10 random colonies confirmed correct targeting of eight colonies, on average, that were positive at both homology arm junctions, and one or two colonies that were only positive at the $5^{\prime}$ homology arm (data not shown). The positive junction PCR at just one of the homology arms may have resulted from a recombination event occurring at the critical exon sequence instead of at the $3^{\prime}$ homology arm. Hence, while using a pair of sgRNAs flanking the critical exon slightly reduced targeting efficiency for pKOMP-Nxn-900, the efficiency was still at least 2000-fold higher relative to the negative control (Fig. S6c).

After observing that a pair of sgRNAs flanking a critical exon maintains high targeting efficiency in ES cells, we hypothesized that this high targeting efficiency would allow targeting of multiple genes by performing a single injection into zygotes that contains corresponding constructs. We also speculated that by using two sgRNAs flanking critical exons, we could obtain additional null alleles by deleting entire 
exons via NHEJ. To this end, we used two KOMP vectors with floxed exon regions (pKOMP-Lrrk2-900 and pKOMP-Glt8d1-900) and sgRNAs targeting genomic sequences not present in the constructs (Fig. S7a, b). We co-injected both targeting constructs, along with two pairs of sgRNA transcripts and Cas9 protein, in mouse zygotes, which produced nine founder pups. Among them, two of the pups contained a floxed Lrrk2 allele (22\%); one of these pups also had a floxed Gltd81 allele (11\%) (Fig. 4a; Table 2). Copy number analysis by RT-qPCR of the Lrrk2 and Glt8d1 regions in nine of the pups revealed that two of them (22\%) had single-exon deletions for Lrrk2, three (33\%) had single-exon deletions for the Glt8dlcritical region, and one (\#8) had a homozygous exon deletion for Glt8dl (Fig. 4b). We further characterized the deletion alleles by diagnostic PCR spanning the critical exon (Fig. 4c). Not surprisingly, pups with a single-exon deletion by RT-qPCR showed diagnostic PCR bands that confirmed the predicted deletion allele. The absence of any Glt8d1 PCR products in pup \#8 suggested that the deletion may include primer-
A

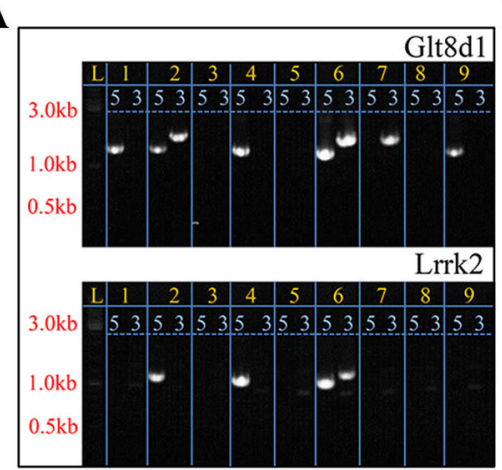

B

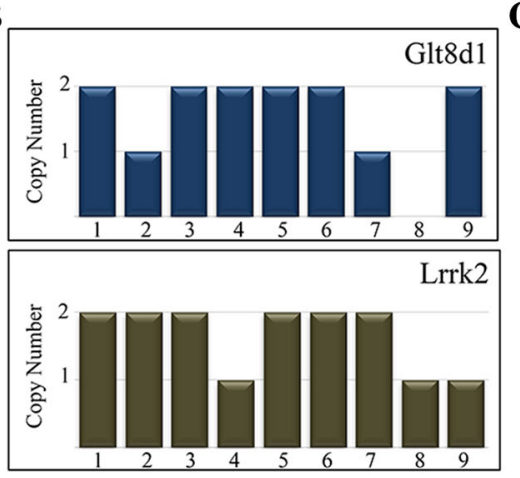

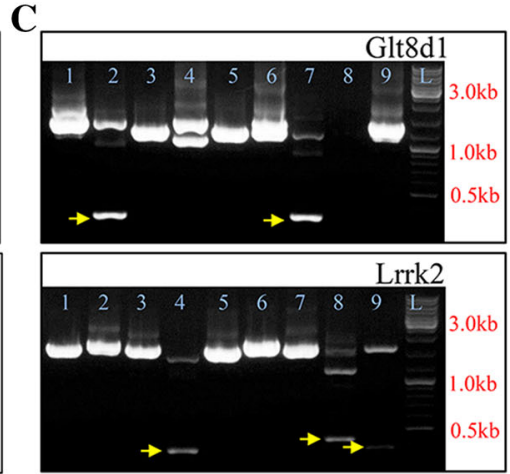

D

\begin{tabular}{|cccccccc|}
\hline PUP ID & PCR & 5'HA & Up & Critical & Down & 3'HA & \\
Glt8d1 & Product & LoxP & sgRNA & Exon & sgRNA & LoxP & Summary \\
\hline 1 & $\sim 1.3 \mathrm{~kb}$ & Yes & na & Yes & Indel & na & 5' LoxP, 3' Indel \\
\hline 2 & $\sim 1.3 \mathrm{~kb}$ & Yes & na & Yes & na & Yes & Targeted \\
& $\sim 0.2 \mathrm{~kb}$ & No & na & Deleted & na & No & CE Deleted \\
3 & $\sim 1.2 \mathrm{~kb}$ & No & indel & Yes & Indel & No & 5' Indel, 3' Indel \\
4 & $\sim 1.3 \mathrm{~kb}$ & Yes & na & Yes & Indel & No & 5' LoxP, 3' Indel \\
& & & & Partially & & & CE Paritially Deleted, \\
& $\sim 1.0 \mathrm{~kb}$ & No & no & Deleted & Indel & No & 3' Indel \\
5 & $\sim 1.2 \mathrm{~kb}$ & No & Indel & Yes & Indel & No & 5' Indel, 3' Indel \\
6 & $\sim 1.3 \mathrm{~kb}$ & Yes & na & Yes & na & Yes & Targeted \\
& $\sim 1.2 \mathrm{~kb}$ & No & Indel & Yes & Indel & No & 5' Indel, 3' Indel \\
7 & $\sim 1.2 \mathrm{~kb}$ & No & Indel & yes & na & Yes & 5' Indel, 3' LoxP \\
& $\sim 1.0 \mathrm{~kb}$ & No & Indel & yes & Indel & No & 5' Indel, 3' Indel \\
& $\sim 0.2 \mathrm{~kb}$ & No & na & Deleted & na & No & CE Deleted \\
\hline 8 & na & na & na & na & na & na & CE Deleted \\
9 & $\sim 1.3 \mathrm{~kb}$ & Yes & na & Yes & Yes & No & 5' LoxP, 3' Indel \\
& $\sim 1.2 \mathrm{~kb}$ & No & Yes & Yes & Yes & No & 5' Indel, 3' Indel \\
\hline
\end{tabular}

Fig. 4 CRISPR/Cas9 induces efficient multi-vector targeting via HDR and NHEJ mediated gene knockout via a single injection to mouse zygotes. a Junction PCR at $5^{\prime}$ and $3^{\prime}$ homology arms to verify targeting of pKOMP-Glt8d1 and pKOMP-Lrrk2 in the nine founder pups. b RT-qPCR to evaluate copy number of Glt8d1 and Lrrk2 critical exons in the nine founder pups. $\mathbf{c}$ PCR to determine critical exon deletion due to sgRNAs targeting regions flanking the critical exons of Gltsd1 or Lrrk2 in the nine founder pups. Yellow arrows, amplicons resulting from critical exon deletions. d, e Summary of sequencing data derived from the gel-extracted PCR products from (c). First columns, ID of pups for Glt8d1 and Lrrk2; second

\begin{tabular}{|c|c|c|c|c|c|c|c|}
\hline $\begin{array}{l}\text { PUP ID } \\
\text { Lrrk2 }\end{array}$ & $\begin{array}{c}\text { PCR } \\
\text { Product }\end{array}$ & $\begin{array}{l}\text { 5'HA } \\
\text { LoxP }\end{array}$ & $\begin{array}{c}\text { Up } \\
\text { sgRNA }\end{array}$ & $\begin{array}{c}\text { Critical } \\
\text { Exon }\end{array}$ & $\begin{array}{r}\text { Down } \\
\text { sgRNA }\end{array}$ & $\begin{array}{l}\text { 3'HA } \\
\text { LoxP }\end{array}$ & Summary \\
\hline 1 & $\sim 1.3 \mathrm{~kb}$ & No & Indel & yes & Indel & No & 5' Indel, 3' Indel \\
\hline 2 & $\sim 1.4 \mathrm{~kb}$ & Yes & na & Yes & Yes & No & 5' LoxP, 3' Indel \\
\hline 3 & $\sim 1.3 \mathrm{~kb}$ & No & Yes & Yes & Yes & No & WT \\
\hline 4 & $\begin{array}{l}\sim 1.2 \mathrm{~kb} \\
\sim 0.2 \mathrm{~kb}\end{array}$ & $\begin{array}{l}\text { Yes } \\
\text { No }\end{array}$ & $\begin{array}{l}\text { na } \\
\text { na }\end{array}$ & $\begin{array}{l}\text { Partially } \\
\text { Deleted } \\
\text { Deleted }\end{array}$ & $\begin{array}{c}\text { Indel } \\
\text { na }\end{array}$ & $\begin{array}{l}\text { No } \\
\text { No }\end{array}$ & $\begin{array}{l}\text { 5' LoxP, } 3 \text { ' Indel } \\
\text { CE Deleted }\end{array}$ \\
\hline 5 & $\sim 1.2 \mathrm{~kb}$ & No & na & $\begin{array}{l}\text { Partially } \\
\text { Deleted }\end{array}$ & na & No & \\
\hline 6 & $\sim 1.4 \mathrm{~kb}$ & Yes & na & $\begin{array}{c}\text { Yes } \\
\text { Partially }\end{array}$ & na & Yes & Targeted \\
\hline & $\sim 1.3 \mathrm{~kb}$ & No & na & Deleted & na & Yes & 3' Indel \\
\hline 7 & $\sim 1.3 \mathrm{~kb}$ & No & Indel & Yes & Yes & No & $5^{\prime}$ Indel \\
\hline 8 & $\sim 1.3 \mathrm{~kb}$ & No & Indel & $\begin{array}{c}\text { Yes } \\
\text { Partially }\end{array}$ & Yes & No & $5^{\prime}$ Indel \\
\hline & $\begin{array}{l}\sim 1.1 \mathrm{~kb} \\
\sim 0.3 \mathrm{~kb}\end{array}$ & $\begin{array}{l}\text { No } \\
\text { No }\end{array}$ & $\begin{array}{l}\text { Indel } \\
\text { na }\end{array}$ & $\begin{array}{l}\text { Deleted } \\
\text { Deleted }\end{array}$ & $\begin{array}{c}\text { Indel } \\
\text { na }\end{array}$ & $\begin{array}{l}\text { No } \\
\text { No }\end{array}$ & $\begin{array}{c}\text { 3' Indel } \\
\text { CE Deleted }\end{array}$ \\
\hline 9 & $\begin{array}{r}\sim 1.3 \mathrm{~kb} \\
\sim 0.2 \mathrm{~kb} \\
\end{array}$ & $\begin{array}{l}\text { No } \\
\text { No }\end{array}$ & $\begin{array}{c}\text { Indel } \\
\text { na }\end{array}$ & $\begin{array}{c}\text { Yes } \\
\text { Deleted }\end{array}$ & $\begin{array}{c}\text { indel } \\
\text { na }\end{array}$ & $\begin{array}{l}\text { No } \\
\text { No }\end{array}$ & $\begin{array}{c}\text { 5' Indel, } 3 \text { ' Indel } \\
\text { CE Deleted }\end{array}$ \\
\hline
\end{tabular}

columns, approximate size of the PCR products that were gel extracted from (c); third columns, indicate presence of $5^{\prime} \mathrm{HA}$ loxP sequences; fourth columns, indicate presence of indels in the Upstream-sgRNA target regions-not applicable (na), indicates presence of $5^{\prime} \mathrm{HA}$ LoxP; fifth columns indicate it the critical exons are present, deleted, or partially deleted; sixth columns, show presence of indels in the Downstream-sgRNA target regions-not applicable (na) indicates presence of $3^{\prime} \mathrm{HA}$ LoxP; seventh columns indicates presence of $3^{\prime} \mathrm{HA}$ loxP sequences; last column, summary of sequence data. Red letters indicate targeted or critical exon (CE) — deleted alleles. (Color figure online) 


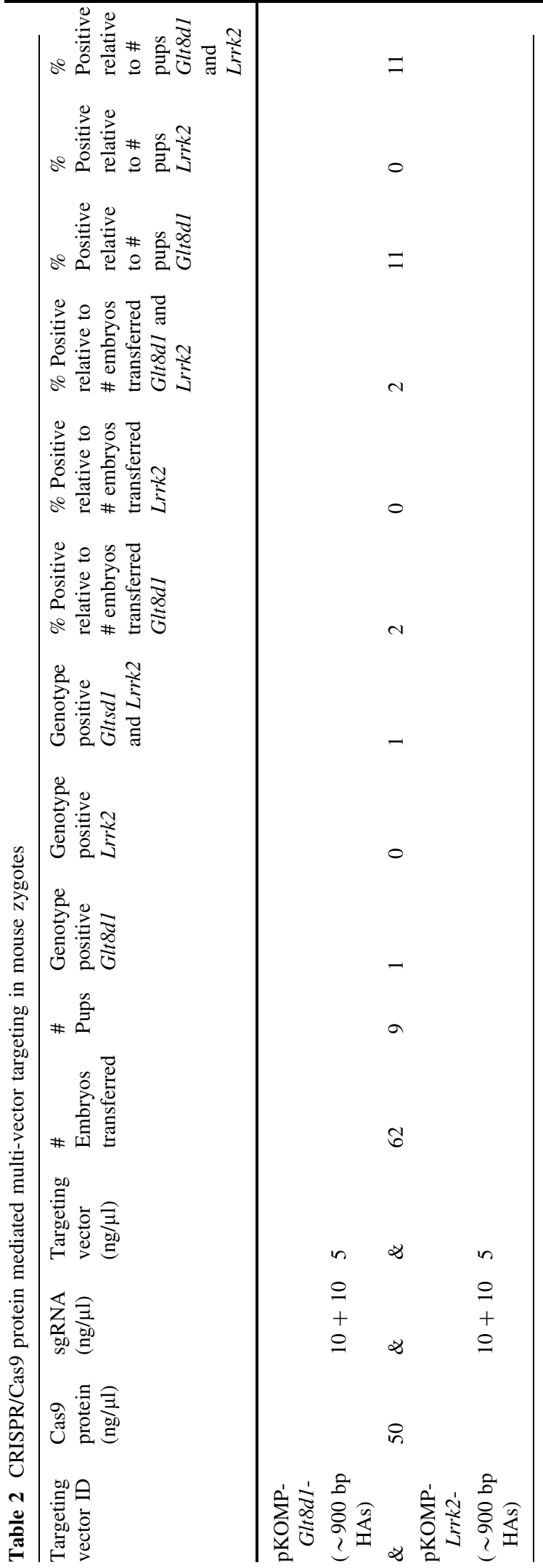

binding sites. Surprisingly, Glt8dl pup \#2 was heterozygous pKOMP-Glt8d1-900, combining the floxed and deletion allele in a single founder. Next, we gel extracted the visible PCR bands, inserted them into a cloning vector, and randomly picked two or three colonies for sequencing. Sequence analysis of Glt8d1 and Lrrk2 (Fig. 4d, e) showed that only one of the nine pups (\#3) was wild type for both Lrrk2 alleles. As such, the overwhelming majority of the targeted alleles had proper construct targeting, had complete or partial deletion of critical exons, and/or obtained indels at the sgRNA target regions. These findings support that using a pair of sgRNAs flanking the critical exon is a highly efficient method for targeting multiple constructs in a single zygote injection experiment, as well as for inducing NHEJ mediated deletion of the critical exon for knocking out gene function in non-targeted alleles.

\section{Discussion}

In this study, we showed that targeting constructs in mouse zygotes with high efficiency is possible through the HDR pathway mediated by the CRISPR/Cas9 system. With a systematic approach, we examined the dsDNA cleavage activity of sgRNAs, determined the role of homology arm length in the targeting constructs, and compared the efficiencies of Cas9, Cas9nickase, and dCas9-FokI in an ES cell model. This approach revealed three interesting findings. First, most of the sgRNA evaluated were highly active. Second, decreasing the homology arm length from approximately $3 \mathrm{kbp}$ to $900 \mathrm{bp}$ did not affect targeting efficiency; however, further decreasing the length and disturbing the symmetry between the two arms considerably decreased the efficiency. Third, while the sgRNA complexed with either Cas9 or Cas9n gave rise to high targeting, switching the endonuclease to dCas9-FokI dramatically lowered the efficiency to a level comparable to the background. After we established the optimal conditions in the ES cell model, we translated them into zygotes with either Cas9 mRNA or protein to further hone and improve the targeting parameters. Similar to our observations in ES cells, we found that the targeting efficiencies in zygotes were comparable between constructs harboring 900 bp or 2 kbp homology arm lengths. Interestingly, we observed higher targeting efficiencies when Cas9 protein was 
used instead of mRNA, similar to that observed by Menoret and colleagues in rat and mouse zygotes (Menoret et al. 2015).

The successful outcome using constructs with 900 bp homology arms and Cas9 protein in zygotes led us to wonder whether we could target multiple vectors in a zygote with a single injection. Here, we designed a new strategy for construct targeting of two different genes for conditional knockout via HDR and deletion of gene function via NHEJ by introducing two pairs of sgRNAs flanking critical exons in a single injection to a zygote. This strategy provided a proof of concept, and for the first time, demonstrated that multiple genes could be targeted for conditional knockout through the HDR pathway and for direct knockout of their functions in non-targeted alleles through a single injection to a zygote. Our findings demonstrate that by systematically optimizing conditions, we established an efficient, robust, and reliable method for construct targeting in zygotes mediated by CRISPR/Cas9.

While developing this study, concerns were raised regarding the possibility that a large fraction of the sgRNAs may possess low cleavage activity, likely requiring each sgRNA to be carefully tested to develop an optimal procedure for construct targeting. Thus, we designed two different assay systems (i.e., the in vitro Cas9 protein assay and the ex vivo SSA assay) for evaluating DNA-cleavage activity. In using these assays, however, we discovered that most of the sgRNAs were highly active at levels comparable to the sgRNA-AAVS1 reported by Mali and colleagues (2013). Based on this result, we speculated that the optimization procedure should shift its focus from evaluating sgRNA activity to minimizing the length of the homology arms to decrease the size of the constructs and to identifying the most efficient type and form of Cas 9 endonucleases. While evaluating the endonucleases, we explored the option of using the obligate dimeric properties of dCas9-FokI to strengthen sgRNA specificity, because some reports raised concerns about high off-target mutation rates induced by CRISPR/Cas9 in human cells (Fu et al. 2013; Hsu et al. 2013; Pattanayak et al. 2013; Lin et al. 2014). Unfortunately, experiments using sgRNA complexed with dCas9-FokI yielded targeting efficiencies comparable to that of background. Many reports suggest that off-target activities may depend on sgRNA target sequences, and that the off-target activities of more promiscuous sgRNAs can be kept at a minimum by engineering them with extra guanines at the $5^{\prime}$ terminus or by choosing unique target sequences near the PAM distal region. This engineering would avoid target sequences with one or two mismatches in other genomic loci (Yang et al. 2013; Cho et al. 2014; Smith et al. 2014; Veres et al. 2014; Kim et al. 2015). Thus, designing sgRNAs with unique target sequences may sufficiently minimize potential off-target effects in animal models.

In addition to a reliable and robust strategy to minimize potential off-target effects, we also need to examine whether conditions described in this study can successfully target more than two constructs in a single injection to a zygote, and whether larger constructs, such as bacterial artificial chromosomes (BACs), can be targeted without losing efficiency. Recently, Yoshimi et al. (2016) described a procedure in which they injected rat zygotes with poly(A) elongated Cas9 mRNA and two sgRNAs and $80 \mathrm{bp}$ ssODNs overlapping the DNA cleavage sites to knockin a $\sim 200 \mathrm{kbp}$ BAC (human SIRPa). With this method, they successfully targeted 1 of 15 founder pups. While they also report that this strategy has the major disadvantage of a high rate of indel mutations at the ssODN mediated conjunction sites, they did not determine whether the strategy could be successfully and reliably reproduced in rat and mouse zygotes.

In summary, we have described an optimized condition for CRISPR/Cas9-mediated construct targeting in mouse zygotes. Our study adds to the growing body of literature describing a myriad of new technological advancements, and, together, they enhance our ability to manipulate the genome. Ultimately, these tools are an essential part of biological sciences, and they facilitate biomedical and translational research toward improving human health.

\section{Experimental procedures}

sgRNA design, expression vectors, and transcription

\section{Design}

sgRNAs were designed using the CRISPR guidedesign tool developed by the Zhang laboratory at MIT (crispr.mit.edu). 
Expression vectors

sgRNA sequences were cloned into U6 target gRNA expression vector as described by Mali et al. (2013).

\section{Transcription}

sgRNA templates were amplified with T7-promotersequence conjugated primers and purified using a PCR cleanup kit (Machery-Nagel). Amplified products were used as templates for transcription using the MEGAshortscript T7 Kit (Thermo Fisher Scientific). Transcripts were purified using the MEGAclear Kit (Thermo Fisher Scientific).

SSA and in vitro cleavage assay

SSA assay

The SSA assay was performed as previously described (Ochiai et al. 2010). Briefly, the target sequence of each sgRNA was cloned into the pGL4-SSA reporter vector and co-tranfected into HEK293T cells with pRL-CMV (Promega) and sgRNA-expressing plasmid. Twenty-four hours post-transfection, firefly and renilla luciferase quantification was done using the Dual-Glo Luciferase Assay Kit (Promega) following the manufacturer's instructions.

\section{In vitro cleavage assay}

The pGL4-SSA plasmid carrying the sgRNA-target sequence $(200 \mathrm{ng}$ ) was incubated with Cas9 protein (500 ng; PNA Bio) and sgRNA transcripts (50 ng) at $37^{\circ} \mathrm{C}$ for $1 \mathrm{~h}$, followed by heat inactivation at $65{ }^{\circ} \mathrm{C}$ for $10 \mathrm{~min}$ and proteinase $\mathrm{K}$ treatment for $30 \mathrm{~min}$ at $60{ }^{\circ} \mathrm{C}$.

\section{KOMP vector modifications}

To modify the homology arm length of KOMP vectors, a two-step approach was used. First, the vector inserts were dissociated from the vector backbone using PacI and AsiSI and cloned into a low copy-vector backbone with a different antibioticresistance marker using Gibson Assembly (NEB). Then, the inserts were gap-repaired into the pUC19 vector backbone with various homology arm lengths.
Cell culture and transfection

\section{ES cell culture}

Mouse JM8.F6 embryonic stem (ES) cells were obtained from the Mouse Biology Program at the University of California, Davis. Cells were maintained as a monolayer on 6-well $\left(9.6 \mathrm{~cm}^{2}\right)$ plates on feeder layers of $\gamma$-irradiated mouse embryonic fibroblasts (MEF) (Global Stem) in Dulbecco's Modified Eagle Medium (DMEM; Thermo Fisher Scientific) supplemented with $15 \%$ fetal bovine serum (FBS; Hyclone), $1000 \mathrm{U} / \mathrm{ml}$ leukemia-inhibitory factor (Millipore), $1 \mathrm{mmol} / \mathrm{l}$ non-essential amino acids (Thermo Fisher Scientific), $2 \mathrm{ml}$ L-glutamine (Thermo Fisher Scientific), and $0.01 \mathrm{mmol} / \mathrm{l}$ 2-mercaptoethanol (Thermo Fisher Scientific).

\section{Electroporation and G418 selection}

On the day of electroporation, ES cells were trypsinized, separated into single cells, and placed in a $37^{\circ} \mathrm{C}$ incubator for $1 \mathrm{~h}$ as a suspension culture in $100 \mathrm{~mm}$ plates coated with $2 \%$ gelatin. Then, $10^{7}$ cells were electroporated using BTX (700 V, $400 \Omega$, $25 \mu \mathrm{F})$ with $15 \mu \mathrm{g}$ Cas9(n) and $15 \mu \mathrm{g} \operatorname{sgRNA}(\mathrm{s})-$ expressing plasmids, along with $15 \mu \mathrm{g}$ of the targeting vector. Electroporated cells were placed on 6-well plates with a monolayer of $\gamma$-irradiated DR4 MEF (Global Stem) feeders. Two days post-transfection, medium was supplemented with $150 \mu \mathrm{g} / \mathrm{ml} \mathrm{G} 418$, and the selection continued for 7-10 days.

Genomic DNA isolation and genotyping

\section{Genomic DNA isolation}

gDNA was isolated from tail biopsies by adding $500 \mu \mathrm{l}$ of lysis buffer $(10 \mathrm{mM}$ Tris, $100 \mathrm{mM} \mathrm{NaCl}$, $10 \mathrm{mM}$ EDTA, $0.5 \%$ SDS) and $20 \mu \mathrm{l}$ of proteinase $\mathrm{K}$ $(20 \mathrm{mg} / \mathrm{ml})$ and incubating them overnight in a $60{ }^{\circ} \mathrm{C}$ water bath. Then, $250 \mu \mathrm{l}$ of $6 \mathrm{M} \mathrm{NaCl}$ was added to each tube, which was centrifuged at $8000 \mathrm{rpm}$ for $10 \mathrm{~min}$ before the supernatant was transferred to new tubes. Isopropanol was added to precipitate the DNA, and $70 \%$ ethanol was used to wash the pellet. 


\section{Genotyping}

Genotyping was done using Sequal Prep Long PCR Kit (Thermo Fisher Scientific). Here, $20 \mathrm{ng}$ of genomic DNA was used as template and amplified following the manufacturer's instructions. All primer sequences are listed in Figure S8.

\section{Real-time quantitative PCR (qPCR)}

Using $20 \mathrm{ng}$ of isolated genomic DNA, qRT-PCR reactions were performed with SYBR Green PCR Master Mix (Applied Biosystems) according to the manufacturer's instructions. Here, $200 \mathrm{nM}$ of each primer pair were used to detect critical exons of Glt8d1 or Lrrk2; Gapdh served as the relative control. Ct values were calculated using Applied Biosystems' SDS2.4 software, and the $\mathrm{Ct}$ values derived from Glt8d1 or Lrrk2 were normalized to the Gapdh gene in the mouse genome to determine the copy number.

Mouse zygote injections

All animal procedures were approved by the Institutional Animal Care and Use Committee at University of California, San Francisco. Super-ovulated female FVB/N mice (4-weeks-old) were mated to FVB/N stud males, and fertilized zygotes were collected from oviducts. Cas9, sgRNA, and plasmid vectors were mixed and injected into the pronucleus of fertilized zygotes. The concentrations of Cas9 protein, Cas9 mRNA, sgRNAs, and plasmid vectors are described in Tables 1 and 2. After the injection procedure, zygotes were implanted into oviducts of pseudopregnant CD1 female mice.

Acknowledgements We extend our gratitude to Chih Chang at the Gladstone Institutes for performing all of the tail biopsies and maintaining the mice. This work was financially supported in part by the BACPAC Resources Center at the UCSF Benioff Children's Hospital Oakland Research Institute and by funding provided by National Institutes of Health (Grant Nos. 5U42OD011175, U42OD012210, 5U24DK092993).

Author contributions C.J.J., D.B.W., K.L., B.R., and P.J.D. developed the conceptual framework. C.J.J., B.R., and P.J.D. designed experiments. C.J.J. and P.J.D. analyzed the data. C.J.J., J.Z., and E.T. performed experiments. C.J.J. and P.J.D. wrote the manuscript.

\section{Compliance with ethical standards}

Conflict of interest We do not have any financial, personal, or professional interests that could be construed to have influenced this manuscript.

Open Access This article is distributed under the terms of the Creative Commons Attribution 4.0 International License (http:// creativecommons.org/licenses/by/4.0/), which permits unrestricted use, distribution, and reproduction in any medium, provided you give appropriate credit to the original author(s) and the source, provide a link to the Creative Commons license, and indicate if changes were made.

\section{References}

Aida T, Chiyo K, Usami T, Ishikubo H, Imahashi R, Wada Y, Tanaka KF, Sakuma T, Yamamoto T, Tanaka K (2015) Cloning-free CRISPR/Cas system facilitates functional cassette knock-in in mice. Genome Biol 16:87

Austin CP, Battey JF, Bradley A, Bucan M, Capecchi M, Collins FS, Dove WF, Duyk G, Dymecki S, Eppig JT, Grieder FB, Heintz N, Hicks G, Insel TR, Joyner A, Koller BH, Lloyd KC, Magnuson T, Moore MW, Nagy A, Pollock JD, Roses AD, Sands AT, Seed B, Skarnes WC, Snoddy J, Soriano P, Stewart DJ, Stewart F, Stillman B, Varmus H, Varticovski L, Verma IM, Vogt TF, von Melchner H, Witkowski J, Woychik RP, Wurst W, Yancopoulos GD, Young SG, Zambrowicz B (2004) The knockout mouse project. Nat Genet 36(9):921-924

Bibikova M, Beumer K, Trautman JK, Carroll D (2003) Enhancing gene targeting with designed zinc finger nucleases. Science 300(5620):764

Boch J (2011) TALEs of genome targeting. Nat Biotechnol 29(2):135-136

Bradley A, Anastassiadis K, Ayadi A, Battey JF, Bell C, Birling MC, Bottomley J, Brown SD, Burger A, Bult CJ, Bushell W, Collins FS, Desaintes C, Doe B, Economides A, Eppig JT, Finnell RH, Fletcher C, Fray M, Frendewey D, Friedel RH, Grosveld FG, Hansen J, Herault Y, Hicks G, Horlein A, Houghton R, Hrabe de Angelis M, Huylebroeck D, Iyer V, de Jong PJ, Kadin JA, Kaloff C, Kennedy K, Koutsourakis M, Lloyd KC, Marschall S, Mason J, McKerlie C, McLeod MP, von Melchner H, Moore M, Mujica AO, Nagy A, Nefedov M, Nutter LM, Pavlovic G, Peterson JL, Pollock J, Ramirez-Solis R, Rancourt DE, Raspa M, Remacle JE, Ringwald M, Rosen B, Rosenthal N, Rossant J, Ruiz Noppinger P, Ryder E, Schick JZ, Schnutgen F, Schofield P, Seisenberger C, Selloum M, Simpson EM, Skarnes WC, Smedley D, Stanford WL, Stewart AF, Stone K, Swan K, Tadepally H, Teboul L, Tocchini-Valentini GP, Valenzuela D, West AP, Yamamura K, Yoshinaga Y, Wurst W (2012) The mammalian gene function resource: the International Knockout Mouse Consortium. Mamm Genome 23(9-10):580-586

Capecchi MR (2005) Gene targeting in mice: functional analysis of the mammalian genome for the twenty-first century. Nat Rev Genet 6(6):507-512 
Cermak T, Doyle EL, Christian M, Wang L, Zhang Y, Schmidt C, Baller JA, Somia NV, Bogdanove AJ, Voytas DF (2011) Efficient design and assembly of custom TALEN and other TAL effector-based constructs for DNA targeting. Nucleic Acids Res 39(12): 82

Cho SW, Kim S, Kim JM, Kim JS (2013) Targeted genome engineering in human cells with the Cas9 RNA-guided endonuclease. Nat Biotechnol 31(3):230-232

Cho SW, Kim S, Kim Y, Kweon J, Kim HS, Bae S, Kim JS (2014) Analysis of off-target effects of CRISPR/Casderived RNA-guided endonucleases and nickases. Genome Res 24(1):132-141

Christian M, Cermak T, Doyle EL, Schmidt C, Zhang F, Hummel A, Bogdanove AJ, Voytas DF (2010) Targeting DNA double-strand breaks with TAL effector nucleases. Genetics 186(2):757-761

Chu VT, Weber T, Graf R, Sommermann T, Petsch K, Sack U, Volchkov P, Rajewsky K, Kuhn R (2016) Efficient generation of Rosa26 knock-in mice using CRISPR/Cas9 in C57BL/6 zygotes. BMC Biotechnol 16(1):4

Cong L, Ran FA, Cox D, Lin S, Barretto R, Habib N, Hsu PD, Wu X, Jiang W, Marraffini LA, Zhang F (2013) Multiplex genome engineering using CRISPR/Cas systems. Science 339(6121):819-823

Fu Y, Foden JA, Khayter C, Maeder ML, Reyon D, Joung JK, Sander JD (2013) High-frequency off-target mutagenesis induced by CRISPR-Cas nucleases in human cells. Nat Biotechnol 31(9):822-826

Gaj T, Gersbach CA, Barbas CF 3rd (2013) ZFN, TALEN, and CRISPR/Cas-based methods for genome engineering. Trends Biotechnol 31(7):397-405

Gondo Y (2008) Trends in large-scale mouse mutagenesis: from genetics to functional genomics. Nat Rev Genet 9(10): 803-810

Guilinger JP, Thompson DB, Liu DR (2014) Fusion of catalytically inactive Cas9 to FokI nuclease improves the specificity of genome modification. Nat Biotechnol 32(6): $577-582$

Horvath P, Barrangou R (2010) CRISPR/Cas, the immune system of bacteria and archaea. Science 327(5962): $167-170$

Hsu PD, Scott DA, Weinstein JA, Ran FA, Konermann S, Agarwala V, Li Y, Fine EJ, Wu X, Shalem O, Cradick TJ, Marraffini LA, Bao G, Zhang F (2013) DNA targeting specificity of RNA-guided Cas9 nucleases. Nat Biotechnol 31(9):827-832

Jiang W, Marraffini LA (2015) CRISPR-Cas: new tools for genetic manipulations from bacterial immunity systems. Annu Rev Microbiol 69:209-228

Jinek M, Chylinski K, Fonfara I, Hauer M, Doudna JA, Charpentier E (2012) A programmable dual-RNA-guided DNA endonuclease in adaptive bacterial immunity. Science 337(6096):816-821

Kim YG, Cha J, Chandrasegaran S (1996) Hybrid restriction enzymes: zinc finger fusions to Fok I cleavage domain. Proc Natl Acad Sci USA 93(3):1156-1160

Kim D, Bae S, Park J, Kim E, Kim S, Yu HR, Hwang J, Kim JI, Kim JS (2015) Digenome-seq: genome-wide profiling of CRISPR-Cas9 off-target effects in human cells. Nat Methods 12(3):237-243 (231 p following 243)
Lee AY, Lloyd KC (2014) Conditional targeting of Ispd using paired Cas9 nickase and a single DNA template in mice. FEBS Open Bio 4:637-642

Lin Y, Cradick TJ, Brown MT, Deshmukh H, Ranjan P, Sarode N, Wile BM, Vertino PM, Stewart FJ, Bao G (2014) CRISPR/Cas9 systems have off-target activity with insertions or deletions between target DNA and guide RNA sequences. Nucleic Acids Res 42(11):7473-7485

Maeder ML, Thibodeau-Beganny S, Osiak A, Wright DA, Anthony RM, Eichtinger M, Jiang T, Foley JE, Winfrey RJ, Townsend JA, Unger-Wallace E, Sander JD, Muller-Lerch F, Fu F, Pearlberg J, Gobel C, Dassie JP, Pruett-Miller SM, Porteus MH, Sgroi DC, Iafrate AJ, Dobbs D, McCray PB Jr, Cathomen T, Voytas DF, Joung JK (2008) Rapid “opensource" engineering of customized zinc-finger nucleases for highly efficient gene modification. Mol Cell 31(2):294-301

Mali P, Esvelt KM, Church GM (2013a) Cas9 as a versatile tool for engineering biology. Nat Methods 10(10):957-963

Mali P, Yang L, Esvelt KM, Aach J, Guell M, DiCarlo JE, Norville JE, Church GM (2013b) RNA-guided human genome engineering via Cas9. Science 339(6121):823-826

Marraffini LA, Sontheimer EJ (2010) CRISPR interference: RNA-directed adaptive immunity in bacteria and archaea. Nat Rev Genet 11(3):181-190

Menoret S, De Cian A, Tesson L, Remy S, Usal C, Boule JB, Boix C, Fontaniere S, Creneguy A, Nguyen TH, Brusselle L, Thinard R, Gauguier D, Concordet JP, Cherifi Y, Fraichard A, Giovannangeli C, Anegon I (2015) Homologydirected repair in rodent zygotes using Cas9 and TALEN engineered proteins. Sci Rep 5:14410

Ochiai H, Fujita K, Suzuki K, Nishikawa M, Shibata T, Sakamoto N, Yamamoto T (2010) Targeted mutagenesis in the sea urchin embryo using zinc-finger nucleases. Genes Cells 15(8):875-885

Pattanayak V, Lin S, Guilinger JP, Ma E, Doudna JA, Liu DR (2013) High-throughput profiling of off-target DNA cleavage reveals RNA-programmed Cas9 nuclease specificity. Nat Biotechnol 31(9):839-843

Ran FA, Hsu PD, Lin CY, Gootenberg JS, Konermann S, Trevino AE, Scott DA, Inoue A, Matoba S, Zhang Y, Zhang F (2013) Double nicking by RNA-guided CRISPR Cas9 for enhanced genome editing specificity. Cell 154(6):1380-1389

Renaud JB, Boix C, Charpentier M, De Cian A, Cochennec J, Duvernois-Berthet E, Perrouault L, Tesson L, Edouard J, Thinard R, Cherifi Y, Menoret S, Fontaniere S, de Croze N, Fraichard A, Sohm F, Anegon I, Concordet JP, Giovannangeli C (2016) Improved genome editing efficiency and flexibility using modified oligonucleotides with TALEN and CRISPR-Cas9 nucleases. Cell Rep 14(9):2263-2272

Rouet P, Smih F, Jasin M (1994) Introduction of double-strand breaks into the genome of mouse cells by expression of a rare-cutting endonuclease. Mol Cell Biol 14(12): 8096-8106

Sander JD, Joung JK (2014) CRISPR-Cas systems for editing, regulating and targeting genomes. Nat Biotechnol 32(4):347-355

Shen B, Zhang J, Wu H, Wang J, Ma K, Li Z, Zhang X, Zhang P, Huang X (2013) Generation of gene-modified mice via Cas9/RNA-mediated gene targeting. Cell Res 23(5): 720-723 
Skarnes WC, Rosen B, West AP, Koutsourakis M, Bushell W, Iyer V, Mujica AO, Thomas M, Harrow J, Cox T, Jackson D, Severin J, Biggs P, Fu J, Nefedov M, de Jong PJ, Stewart AF, Bradley A (2011) A conditional knockout resource for the genome-wide study of mouse gene function. Nature 474(7351):337-342

Smith C, Gore A, Yan W, Abalde-Atristain L, Li Z, He C, Wang Y, Brodsky RA, Zhang K, Cheng L, Ye Z (2014) Wholegenome sequencing analysis reveals high specificity of CRISPR/Cas9 and TALEN-based genome editing in human iPSCs. Cell Stem Cell 15(1):12-13

Stanford WL, Cohn JB, Cordes SP (2001) Gene-trap mutagenesis: past, present and beyond. Nat Rev Genet 2(10): 756-768

Sung YH, Kim JM, Kim HT, Lee J, Jeon J, Jin Y, Choi JH, Ban YH, Ha SJ, Kim CH, Lee HW, Kim JS (2014) Highly efficient gene knockout in mice and zebrafish with RNA-guided endonucleases. Genome Res 24(1): 125-131

Testa G, Schaft J, van der Hoeven F, Glaser S, Anastassiadis K, Zhang Y, Hermann T, Stremmel W, Stewart AF (2004) A reliable lacZ expression reporter cassette for multipurpose, knockout-first alleles. Genesis 38(3):151-158

Tsai SQ, Wyvekens N, Khayter C, Foden JA, Thapar V, Reyon D, Goodwin MJ, Aryee MJ, Joung JK (2014) Dimeric CRISPR RNA-guided FokI nucleases for highly specific genome editing. Nat Biotechnol 32(6):569-576

Veres A, Gosis BS, Ding Q, Collins R, Ragavendran A, Brand $\mathrm{H}$, Erdin S, Cowan CA, Talkowski ME, Musunuru K (2014) Low incidence of off-target mutations in individual CRISPR-Cas9 and TALEN targeted human stem cell clones detected by whole-genome sequencing. Cell Stem Cell 15(1):27-30
Wang H, Yang H, Shivalila CS, Dawlaty MM, Cheng AW, Zhang F, Jaenisch R (2013) One-step generation of mice carrying mutations in multiple genes by CRISPR/Casmediated genome engineering. Cell 153(4):910-918

Wang L, Shao Y, Guan Y, Li L, Wu L, Chen F, Liu M, Chen H, Ma Y, Ma X, Liu M, Li D (2015) Large genomic fragment deletion and functional gene cassette knock-in via Cas9 protein mediated genome editing in one-cell rodent embryos. Sci Rep 5:17517

Wiedenheft B, Sternberg SH, Doudna JA (2012) RNA-guided genetic silencing systems in bacteria and archaea. Nature 482(7385):331-338

Yang H, Wang H, Shivalila CS, Cheng AW, Shi L, Jaenisch R (2013) One-step generation of mice carrying reporter and conditional alleles by CRISPR/Cas-mediated genome engineering. Cell 154(6):1370-1379

Yang H, Wang H, Jaenisch R (2014) Generating genetically modified mice using CRISPR/Cas-mediated genome engineering. Nat Protoc 9(8):1956-1968

Yoshimi K, Kaneko T, Voigt B, Mashimo T (2014) Allelespecific genome editing and correction of disease-associated phenotypes in rats using the CRISPR-Cas platform. Nat Commun 5:4240

Yoshimi K, Kunihiro Y, Kaneko T, Nagahora H, Voigt B, Mashimo T (2016) ssODN-mediated knock-in with CRISPR-Cas for large genomic regions in zygotes. Nat Commun 7:10431

Yu Y, Bradley A (2001) Engineering chromosomal rearrangements in mice. Nat Rev Genet 2(10):780-790

Zhou H, Liu B, Weeks DP, Spalding MH, Yang B (2014) Large chromosomal deletions and heritable small genetic changes induced by CRISPR/Cas9 in rice. Nucleic Acids Res 42(17):10903-10914 